\title{
Genomic organization and localization of the NAD-dependent histone deacetylase gene sirtuin 3 (Sirt3) in the mouse
}

\author{
ULRICH MAHLKNECHT and SUSANNE VOELTER-MAHLKNECHT \\ Saarland University Medical Center, Department of Internal Medicine, Division of Immunotherapy and \\ Gene Therapy, José Carreras Research Center, Kirrberger Str., D-66421 Homburg/Saar, Germany
}

Received September 9, 2010; Accepted October 17, 2010

DOI: $10.3892 /$ ijo.2010.872

\begin{abstract}
Sirtuin 3 (SIRT3) is a nicotinamide adenine dinucleotide $\left(\mathrm{NAD}^{+}\right)$-dependent deacetylase, which belongs to the Silent information regulator 2 (Sir2) family of histone deacetylases (sirtuin HDACs). The yeast Sir2 protein and its mammalian derivatives play a central role in epigenetic gene silencing, DNA repair and recombination, cell-cycle, microtubule organization, and in the regulation of aging. We have isolated and characterized the murine Sirt3 genomic sequence, which spans a region of 18,646 bp and which has one single genomic locus. Determination of the exon-intron splice junctions identified murine SIRT3 to be encoded by 7 exons ranging in size from 101 (exon 4) to 420 bp (exon 7). Characterization of the $5^{\prime}$ flanking genomic region, which precedes the murine Sirt3 open reading frame, revealed a number of STATx, GATA and SP1 transcription factor binding sites. A CpG island was not detected. The 1,473-bp murine Sirt3 transcript has an open reading frame of $774 \mathrm{bp}$ and encodes a 257-aa protein (cytoplasmic SIRT3) with a predictive molecular weight of $28.8 \mathrm{kDa}$ and an isoelectric point of 5.82. Recently, a 1,406-bp murine SIRT3 splice variant that encodes a 334-aa mitochondrial precursor protein with a molecular weight of $36.6 \mathrm{kDa}$ and an isoelectric point of 7.19 has been described. Fluorescence in situ hybridization analysis identified a single genomic locus for murine Sirt3 gene on chromosome 7F4 and which is neighbored by the Ric8 and PSMD13 genes. Our study brings light and a number of corrections and additions to previous reports on the genomic organization and the genomic sequence of
\end{abstract}

Correspondence to: Dr U. Mahlknecht, Saarland University Medical Center, Department of Internal Medicine, Division of Immunotherapy and Gene Therapy, José Carreras Research Center, Kirrberger Str., Bldg. 45.3, D-66421 Homburg/Saar, Germany E-mail: ulrich.mahlknecht@uks.eu

Abbreviations: HDAC, histone deacetylase; HAT, histone acetyltransferase

Key words: sirtuins, histones, chromatin, histone deacetylase, chromosomes, genes, structural murine Sirt3, which may be of importance in view of studies on potential genetic polymorphisms in relation to cellular respiration, metabolism, aging-related disease and cancer.

\section{Introduction}

Mammalian histone deacetylases (HDACs) catalyze the removal of acetyl groups from the a-amino group of lysine residues. Based on phylogenetic analyses, structural and functional similarities, HDACs are grouped into four subclasses, which comprise three classes of non-sirtuin HDACs, i.e., the yeast histone deacetylase RPD3 homologs (class I HDACs), the yeast histone deacetylase HDA1 homologs (class II HDACs), HDAC11 as the only class IV HDAC, and the sirtuins (class III HDACs), which received their name based on their homology with the yeast Sir2 protein (1-3). The main distinguishing feature, that discriminates the sirtuins from other HDACs, is their exceptional enzymatic mechanism. While class I, II and IV HDACs are $\mathrm{Zn}^{2+}$-dependent hydrolases, the sirtuins possess a unique NAD-dependent deacetylase activity and as shown for a few sirtuins, a secondary ADPribosyltransferase activity $(4,5)$. To date, seven mammalian sirtuins have been identified (SIRT1-SIRT7), which share one common Sir2 catalytic domain $(2,6-8)$. This deacetylase domain is being flanked by highly variable $\mathrm{N}$-terminal and $\mathrm{C}$-terminal extensions, suggesting the acquisition of new functions through evolution, which may be required for the regulation of their subcellular localization and/or catalytic activity $(8,9)$. While SIRT1 may be found both, in cell nucleus and in the cytoplasm, SIRT2 has been reported to be exclusively cytoplasmic. SIRT3, SIRT4 and SIRT5 have been reported to contain $\mathrm{N}$-terminal mitochondrial targeting sequences and have therefore been suggested to localize to the matrix of mitochondria (10-16), while SIRT6 and SIRT7 are solely found in the cell nucleus (17). SIRT3 is the mitochondrial sirtuin that exhibits the highest degree of sequence conservation with SIRT1, and is the relatively best-understood mitochondrial sirtuin so far. Recently, a novel SIRT3 splice variant was reported, which contains 2 translational start sites upstream of the originally reported start site (18). These longer 334-aa Sirt3 splice variants have been reported to localize to the mitochondria, while the shorter, originally reported 257-aa Sirt3 represents the processed forms of these longer variants, which does in fact not localize to the mitochondria, but to the cytoplasm (18) and may translocate 
to the nucleus during cellular stress $(17,18)$. Mouse SIRT3 is strongly expressed in tissues that are highly metabolically active such as brown adipose tissue, muscle, liver, the kidneys, heart and brain $(13,19,20)$. SIRT3 null mice do not have an obvious metabolic or developmental phenotype (21). SIRT3 levels are increased in the context of calorie restriction, stress, exercise $(20,22,23)$ and in some forms of cancer, such as node-positive breast cancer (24).

SIRT3 binds and deacetylates at least three metabolic mitochondrial substrates such as acetyl-CoA-synthetase (AceCS) (14,25), complex I and glutamate dehydrogenase (GDH) and interestingly, SIRT3-driven deacetylation appears to activate the enzymatic activity of these proteins $(21,26)$. SIRT3 activates the nuclear transcription of mitochondriarelated genes such as UCP1 (uncoupling protein 1), PGC-1 $\alpha$ (peroxisome proliferator-activated receptor $\gamma$ ) and COX (cytochrome oxidase) IV and V, which activate CREB (20). Also, SIRT3 regulates the mitochondrial protein synthesis through deacetylation of the mitochondrial ribosomal protein MRPL10 (27). SIRT3 is tightly linked to mitochondrial metabolism, respiration (28) and the regulation of human life span $(29,30)$.

Based on the fact that the mitochondria are essential for a sufficient energy supply to the cell, the mitochondrial homeostasis is crucially important as to cell survival. Mitochondrial dysfunction may be associated with numerous metabolic diseases, degenerative disorders and cancer. Based on the multifaceted mitochondrial functions of SIRT3, SIRT3 polymorphisms could be of pathophysiological significance in this context.

\section{Materials and methods}

Identification of the murine Sirt3 cDNA. Homology searches of the EST database at NCBI (National Center for Biotechnology Information) with the yeast SIR2 protein sequence (GenPept P06700) yielded 10 mRNA sequences of variable length (GenBank AF299338.1, AF299339.1, AK075861.1, AK086910.1, BC025878.1, BF681854.1, CB953804.1, CT010402.1, EU886466.1 and FJ621493.1), of which mRNA sequence AF299338.1, which contained the murine Sirt3 mRNA reference sequence was then used for the identification of the murine Sirt3 genomic clone.

Identification of BAC genomic clone RP23-114A6. The murine Sirt3 genomic clone was identified from a murine BAC genomic library (RZPD, Berlin, Germany) after in silico screening with the Sirt3 cDNA (GenBank clone AF299338.1), which was shown to contain the full-length murine Sirt3 cDNA. BAC clone RP23-114A6 was identified to contain an insert with a size of $\sim 120 \mathrm{~kb}$ in the vector pBACe3.6, which included the murine Sirt3 genomic sequence. BAC genomic DNA was prepared according to published protocols (31) and the murine Sirt3 insert was confirmed by cycle sequencing (32).

Instrumental methods. Dye terminator cycle sequencing was performed using the ABI PRISM BigDye ${ }^{\mathrm{TM}}$ Terminator Cycle Sequencing Ready Reaction Kit with AmpliTaq ${ }^{\mathrm{TM}}$ DNA polymerase (Perkin-Elmer, Branchburg, NJ) and analyzed with an ABI PRISM 310 Genetic Analyzer which utilizes the four-color sequencing chemistry.

PCR methods. The Sirt1 sequence was partially sequenced by primer walking on both strands using a direct sequencing strategy (32). Sequencing reactions were performed using $0.6 \mu \mathrm{g}$ cDNA and 20-30mer oligonucleotide primers (Thermo Electron, Dreieich, Germany). Sequencing reactions were set up in a volume of $20 \mu \mathrm{l}$ containing $10 \mathrm{pmol}$ of the sequencing primer, $4 \mu \mathrm{l}$ BigDye Terminator Cycle Sequencing Ready Reaction Mix (Perkin-Elmer, Norwalk, CT), DNA as indicated and $\mathrm{ddH}_{2} \mathrm{O}$ added up to a final volume of $20 \mu \mathrm{l}$. The thermal cycling profile for the sequencing of the cDNA-clones was as follows: denaturation at $95^{\circ} \mathrm{C}$ for $30 \mathrm{sec}$, annealing at $50^{\circ} \mathrm{C}$ for $15 \mathrm{sec}$, extension at $60^{\circ} \mathrm{C}$ for $4 \mathrm{~min}$ ( 25 cycles $)$, and storage at $4^{\circ} \mathrm{C}$.

\section{Chromosomal localization by fluorescence in situ hybridization (FISH)}

Cell culture and chromosome preparation. Standard chromosome preparations were used from a mouse embryonic fibroblast cell line. Slide preparation: In order to remove excess of cytoplasm, slides were treated with pepsin $(0.5 \mathrm{mg} / \mathrm{ml}$ in $0.01 \mathrm{M} \mathrm{HCl}, \mathrm{pH} 2.0$ ) at $37^{\circ} \mathrm{C}$ for $40 \mathrm{~min}$. Slides where then washed $10 \mathrm{~min}$ in $1 \mathrm{X}$ PBS at room temperature followed by an ethanol series $(70,90$ and 100\%) and air-dried. $B A C$ genomic clone $R P 23-114 A 6$, which was shown to contain the murine Sirt3 gene, was used as a probe. Probe labeling: The BAC DNA was labeled by a standard nick translation procedure. Digoxigenin (Roche Diagnostics) was used as labeled dUTP at the concentration of $40 \mu \mathrm{M}$. Probe length was analyzed on a $1 \%$ agarose gel. The probe showed the optimal average length of $\sim 300 \mathrm{bp}$ after nick translation. Hybridization and probe detection: About $50 \mathrm{ng}$ DNA was pooled together with $2 \mu \mathrm{g}$ cot- 1 in $10 \mu \mathrm{l}$ hybridization buffer (50\% formamide, 2 X SSC, $10 \%$ dextran sulfate). The DNA was applied to chromosomes fixed on a slide, mounted with a cover slip and sealed with rubber cement. Probe DNA and chromosomes were denatured together at $72^{\circ} \mathrm{C}$ for $3 \mathrm{~min}$. Hybridization was overnight at $37^{\circ} \mathrm{C}$ in a wet chamber. After hybridization the cover slip was carefully removed and the slide was washed in $2 \mathrm{X}$ SSC for $8 \mathrm{~min}$. Slides were then incubated at $70^{\circ} \mathrm{C}$ in $0.4 \mathrm{X} \mathrm{SSC} / 0.1 \%$ Tween for $1 \mathrm{~min}$. After equilibration in $4 \mathrm{X} \mathrm{SSC} / 0.1 \%$ Tween for $5 \mathrm{~min}$ the rhodamine coupled antibody was applied (dilution of 1-400). Incubation was for $45 \mathrm{~min}$ at $37^{\circ} \mathrm{C}$. The slide was then washed twice in $4 \mathrm{X} \mathrm{SSC} / 0.1 \%$ Tween for $10 \mathrm{~min}$ at $45^{\circ} \mathrm{C}$ followed by staining in DAPI (4',6-Diamidino-2-phenylindole) for $10 \mathrm{~min}$. For microscopy, the slide was mounted in antifade solution (Vectashield). Microscopy: In situ hybridization signals were analyzed on a Zeiss Axioplan II microscope. Each image plain (blue and orange) was recorded separately with a b/w CCD camera. Chromosomes and FISH signals were then displayed in false colors and images merged on the computer. Camera control, image capture and merging were done with SmartCapture X software (Digital Scientific, Cambridge, UK).

Sequence analysis and computer database searches. DNA sequence analysis was performed using the HUSAR 
Table I. Exon/intron splice-junctions of the murine Sirt3 gene: exon sequences are given in uppercase and intron sequences are given in lowercase letters. ${ }^{\mathrm{a}}$

\begin{tabular}{lccccc}
\hline $\begin{array}{l}\text { Exon } \\
\text { no. }\end{array}$ & $\begin{array}{c}\text { Exon } \\
\text { size }(\mathrm{bp})\end{array}$ & 5'-Splice donor & $\begin{array}{c}\text { Intron } \\
\text { no. }\end{array}$ & $\begin{array}{c}\text { Intron } \\
\text { size }(\mathrm{bp})\end{array}$ & 3'-Splice acceptor \\
\hline $\mathbf{1 - A}$ & 152 & CGAGGACTAGgtgtgatgcaaa & $\mathbf{1 - A}$ & $\mathbf{2 8 8}(288)$ & gctcacgggggcGGGGATTCGG \\
$\mathbf{1 - B}$ & 107 & GGTCCGGGAGgtgagccacggc & $\mathbf{1 - B}$ & $\mathbf{3 , 6 1 7}(4 \mathrm{~kb})$ & tcttgctgtcagTGTTACAGGT \\
$\mathbf{2}$ & $189(181)$ & CGGACTTCAGgtatgctgcggt & $\mathbf{2}$ & $\mathbf{7 4}(74)$ & ttctctctgCagATCCCCAGGG \\
$\mathbf{3}$ & 233 & CTTGAGAGAGgtgagcctttgg & $\mathbf{3}$ & $\mathbf{1 , 1 9 5}(1,400)$ & tttgaatttcagCATCTGGGAT \\
$\mathbf{4}$ & 101 & AGACATATGGgtgagtcacctc & $\mathbf{4}$ & $\mathbf{6 , 7 0 3}(7 \mathrm{~kb})$ & ctgattcCCCagGCTGATGTGA \\
$\mathbf{5}$ & 162 & CTCCCTGGAGgtttgttgaaag & $\mathbf{5}$ & $\mathbf{4 , 3 0 4}(4.5 \mathrm{~kb})$ & gttatctttcagGTGGAGCCTT \\
$\mathbf{6}$ & 210 & CTCCCTGGAGgtttgttgaaag & $\mathbf{6}$ & $\mathbf{8 9 1}(1.4 \mathrm{~kb})$ & tgtctgttctagCTGGATGGAC \\
$\mathbf{7}$ & 420 & ACGTGGCAAGgtacagtggctc & & & \\
\hline
\end{tabular}

${ }^{a}$ The sizes of the single exons and introns are indicated. Consensus splice donor and splice acceptor sequences are given in bold. The intron sizes that have been reported by Yang et al are shown in parenthesis, while the intron sizes that we have identified are shown in bold.

Table II. Sequence identity and similarity among class III sirtuin-HDACs in the mouse. ${ }^{a}$

\begin{tabular}{|c|c|c|c|c|c|c|c|c|}
\hline $\begin{array}{l}\text { Identity } \\
\text { similarity }\end{array}$ & $\begin{array}{l}\text { Mouse } \\
\text { SIRT1 }\end{array}$ & $\begin{array}{l}\text { Mouse } \\
\text { SIRT2 }\end{array}$ & $\begin{array}{l}\text { Mouse } \\
\text { SIRT3 }\end{array}$ & $\begin{array}{l}\text { Mouse } \\
\text { SIRT4 }\end{array}$ & $\begin{array}{l}\text { Mouse } \\
\text { SIRT5 }\end{array}$ & $\begin{array}{l}\text { Mouse } \\
\text { SIRT6 }\end{array}$ & $\begin{array}{l}\text { Mouse } \\
\text { SIRT7 }\end{array}$ & $\begin{array}{l}\text { Yeast } \\
\text { SIR2 }\end{array}$ \\
\hline Mouse SIRT1 & & 41 & 42 & 31 & 27 & 23 & 23 & 42 \\
\hline Mouse SIRT2 & 59 & & 51 & 29 & 26 & 27 & 27 & 30 \\
\hline Mouse SIRT3 & 64 & 66 & & 29 & 30 & 32 & 28 & 38 \\
\hline Mouse SIRT4 & 48 & 46 & 43 & & 29 & 27 & 27 & 28 \\
\hline Mouse SIRT5 & 41 & 44 & 45 & 48 & & 23 & 23 & 28 \\
\hline Mouse SIRT6 & 40 & 42 & 45 & 42 & 38 & & 41 & 24 \\
\hline Mouse SIRT7 & 41 & 45 & 43 & 43 & 37 & 55 & & 23 \\
\hline Yeast SIR2 & 59 & 48 & 53 & 46 & 42 & 40 & 40 & \\
\hline
\end{tabular}

aThe indicated numbers represent the percentage of sequence identity and similarity from pairwise sequence comparisons.

(Heidelberg Unix Sequence Analysis Resources) server hosted by the Biocomputing Service Group at the German Cancer Research Center (DKFZ, Heidelberg, Germany) and the UniGene and LocusLink programs at the National Center for Biotechnology Information (NCBI). Sequence comparisons were performed with the BLAST algorithm of the GenBank and EMBL databases (33). Protein similarity scores were calculated from fast alignments generated by the method of Wilbur and Lipman with the CLUSTAL W Multiple Alignment Program Version 1.7 and with the BLAST algorithm at NCBI (Table II) (34). Protein motifs were identified online at the ExPASy (Expert Protein Analysis System) proteomics server of the Swiss Institute of Bioinformatics (SIB) with the program PROSITE and double-checked using the MotifFinder program hosted by the GenomeNet WWW server at Institute for Chemical Research, Kyoto University (Japan), but still remain to be experimentally confirmed. Potential transcription factor binding sites were identified with the TRANSFAC program, which is part of the GenomeNet Computation Service, which is hosted by the Bioinformatics Center at the Institute for Chemical Research at the Kyoto University. Sequence similarities were calculated with the GAP software, which considers all possible alignments and gap positions between two sequences and creates a global alignment that maximizes the number of matched residues and minimizes the number and size of gaps on the HUSAR server. Repetitive elements were identified on the Repeat Masker Server at the University of Washington and $\mathrm{CpG}$ elements were found with the EMBOSS CPG software hosted by the European Bioinformatics Institute (EMBL outstation) (Figs. 1 and 2).

Phylogenetic analysis. Phylogenetic trees were constructed from the known murine class III histone deacetylase sequences, which were obtained from a protein sequence similarity 
A

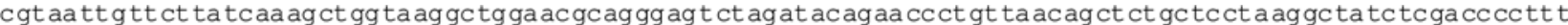

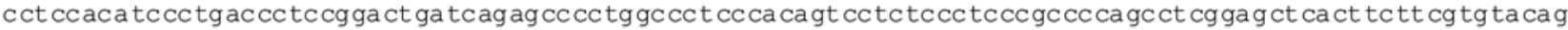
ctctt cca aa cggt gcca aa cggcag cctggeccgg cccgga gct ct ga ct ct gctg ta agaagg ccgg ca ca tcct tca tgat tg caga gagctg gga a ca acaca ag ca ct aa gcag tcag aa cc gg ca gg ga tg gcgcacgga ag tgct cgct ca ct tccg gcaccg cg gg cg cg cgg gg ca tg ct gg ga gcgt c

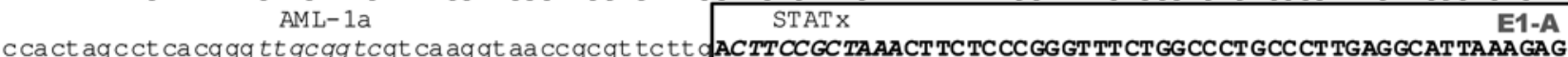

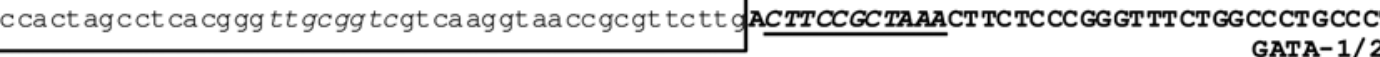

TAGAGGT GCCTGGGGACA GC TAGCCACT GACTGGTCACGTAGCCTCAAGCC TGCAGACT TGGGTCCTCT GAAA CCGGATGGCGT TT GGCGAGGACTAG

tgt ga tgcaa at cg ct cagagagcat tagt ta tcacccgcct act ca aggagg tcgagagcgg cg ta aa acga tgaa at ctcccgg tt tg gctt cccaa $\mathrm{AP}-1$

Spl 1

ccalaagtcagag tcccacagagggagaagctgccagcaccaggcataaaca cagcct tccacact tcgggt ta tcgtgcgggggctgggctggg ctc Sp1

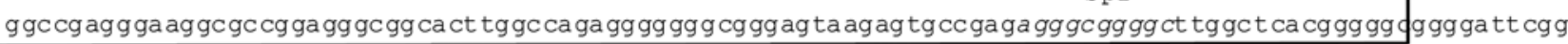

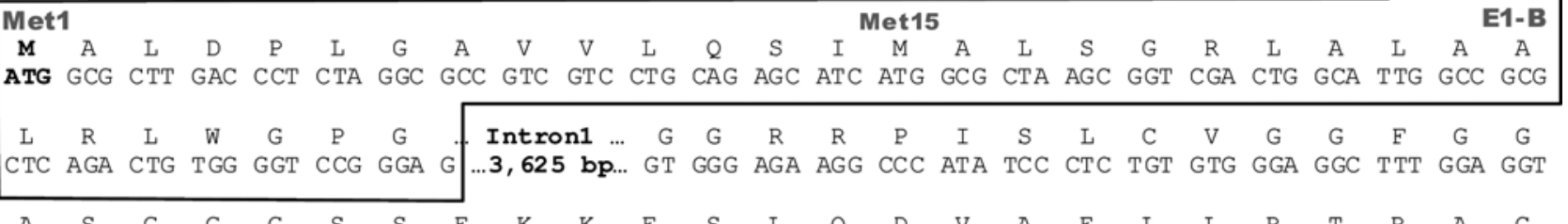

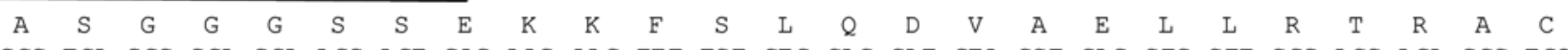
GCC TCA GGC GGA GGA AGC AGT GAg AAg AAg TTT TCT CTG CAg GAT GTA GCT GAG CTG CTT CGG ACC AGA GCC TGC Met78

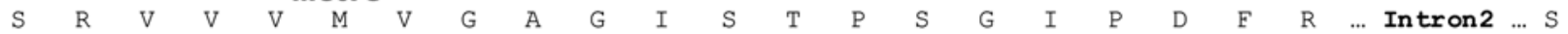
Agt AGG GTG GTG GTC ATG GTG GGG GCC GGC ATC AGC ACA CCC AGT GGC ATC CCG GAC TTC AG ... 74 bp ... A TCC

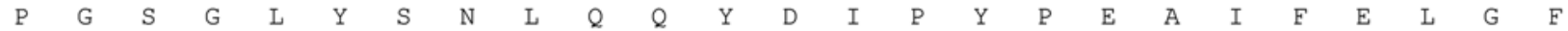
CCA GGG AGC GGC CTC TAC AGC AAC CTT CAG CAG TAT GAC ATC CCG TAC CCT GAA GCC ATC TTT GAA CTT GGC TTT $\begin{array}{lllllllllllllllllllllllllllllll}\mathrm{F} & \mathrm{F} & \mathrm{H} & \mathrm{N} & \mathrm{P} & \mathrm{K} & \mathrm{P} & \mathrm{F} & \mathrm{F} & \mathrm{M} & \mathrm{L} & \mathrm{A} & \mathrm{K} & \mathrm{E} & \mathrm{L} & \mathrm{Y} & \mathrm{P} & \mathrm{G} & \mathrm{H} & \mathrm{Y} & \mathrm{R} & \mathrm{P} & \mathrm{N} & \mathrm{V} & \mathrm{T}\end{array}$ TTC TTT CAC AAC CCC AAG CCC TTT TTC ATG TTG GCC AAG GAG CTG TAC CCT GGG CAC TAC AGG CCC AAT GTC ACT

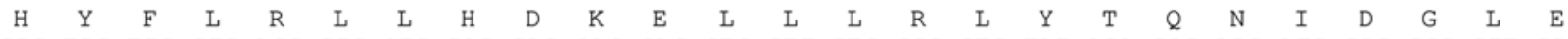
CAC TAC TTC CTG AGG CTC CTC CAC GAC AAG GAG CTG CTT CTG CGG CTC TAT ACA CAG AAC ATC GAC GGG CTT GAG $\begin{array}{llllllllllllllllllllllllllll}R & \text {... Intron3 } & \ldots & \mathrm{A} & \mathrm{S} & \mathrm{G} & \mathrm{I} & \mathrm{P} & \mathrm{A} & \mathrm{S} & \mathrm{K} & \mathrm{L} & \mathrm{V} & \mathrm{E} & \mathrm{A} & \mathrm{H} & \mathrm{G} & \mathrm{T} & \mathrm{F} & \mathrm{V} & \mathrm{T} & \mathrm{A} & \mathrm{T}\end{array}$ AGA G ... 1,195 bp ... CA TCT GGG ATC CCT GCC TCA AAG CTG GTT GAA GCC CAC GGG ACC TTT GTA ACA GCT ACA

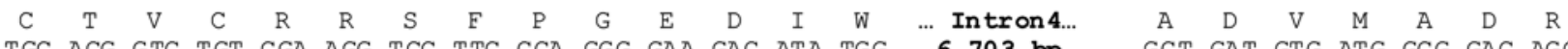
TGC ACG GTC TGT CGA AGG TCC TTC CCA GGG GAA GAC ATA TGG ... 6, 703 bp ... GCT GAT GTG ATG GCG GAC AGG

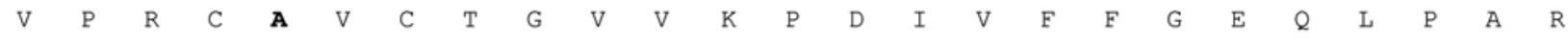
GTG CCC CGC TGC GCT GTC TGT ACT GGC GTT GTG AAA CCC GAC ATT GTG TTC TTT GGG GAG CAG CTG CCT GCA AGG

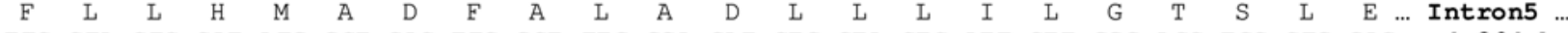
TTC CTA CTC CAT ATG GCT GAC TTC GCT TTG GCA GAT CTG CTA CTC ATT CTT GGG ACC TCC CTG GAG ... 4,304 bp

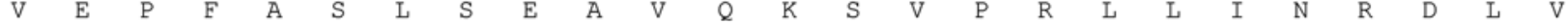
GTG GAG CCT TTT GCC AGC TTG TCT GAA GCA GTA CAG AAA TCA GTG CCC CGA CTG CTC ATC AAT CGA GAC TTG GTG

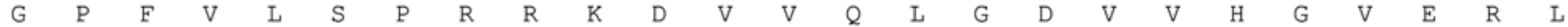
GgG CCG TTC GTT CTG AGT CCT CGA AGg AAA GAT GTG GTC CAG CTA GGg GAT GTA GTT CAT GGT GTG GAA AGg CTG

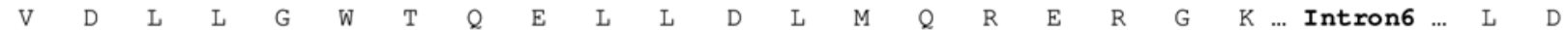
GTG GAC CTC CTG GGG TGG ACA CAA GAA CTG CTG GAT CTT ATG CAG CGg GAA CGT GGC AAG ... 891 bp ... CTG GAT G Q D $R$ * GGA CAG GAC AGA TAA gactatggcttcttcacctggggaagtcacacagcagatcatcctatgtccagcaagacttcatgcctgaagacagctc caa ca cg t tt acaa acatga acca ga ccacaa ca tg tg gcctgga cagt gg tcct cc ga gg ctgc ct tt ggaa aggctga ccagggat gt ctacccttg gggcccctccatgtgtgcgccctgtccacctcatcactgctgaaggtgtagtgcaggtgctgctt tctgcagcggcc t aagt tatcacgagggcagc a ca gcacg cc cg tcgccagg cagg cgat gcactagg gcaa tctagca tg ttga tcgg ta aagtggca tctt ta acta ca a ca tcat tt ct tgca tg aaa

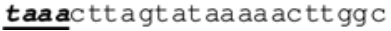

Figure 1. The complete sequence of the murine Sirt3 cDNA together with the predicted amino acid sequence is shown with the location of each intron with respect to the cDNA sequence. (A) 1,406 bp murine SIRT3 splice variant encoding a 334-aa mitochondrial precursor protein with a molecular weight of $36.6 \mathrm{kDa}$ and an isoelectric point of 7.19 (18). Within exon 1B (E 1-B) translation may start in position Met1 or Met15.

search with the yeast SIR2 protein using the BLAST 2.0 program at NCBI database (Non-redundant GenBank CDS: translations $+\mathrm{PDB}+$ SwissProt + SPupdate $+\mathrm{PIR})$. Progressive multiple sequence alignments were performed with the CLUSTAL W Multiple Alignment Program Version 1.7 (35). Trees were calculated and drawn with the PUZZLE software, which constructs phylogenetic trees from molecular sequence data and which is hosted by the HUSAR (Heidelberg Unix Sequence Analysis Resources) server from the Biocomputing Service Group at the German Cancer Research Center (DKFZ) and displayed with the PHYLIP2TREE program. While vertical numbers along branches represent percentage values 
$\mathbf{B}$

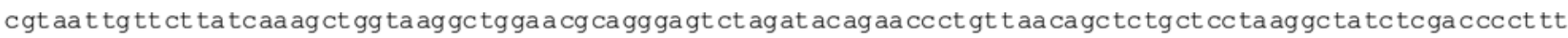

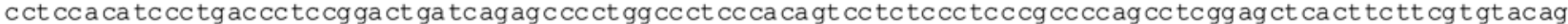
ctctt cca aa cg gtgcca aa cg gcag cctggcccggcccggag ct ct ga ct ct gctg taagaaggc cg gcacat cc tt ca tgat tgcagagagctgg ga a ca aca ca ag ca ct aa gcag tcagaa cc ggcagggat gg cgca cg ga ag tgct cg ct ca ct tccggca cc gc gg gcgcgcgg gg ca tgct gggagcg tc AML-1a STATX c ca ct a gc ct ca cggg ttgcggtcgt ca ag gt aa c cg g t t ct to ACTTCCGCTAAACTTCTCCCGGGTTTCTGGCCCTGCCCT TGAGGCATTAAAGAG GATA-1/2

TAGAGGTGCCTGGGGACA GCTAGCCACT GACTGGTCACGTAGCCTCAAGCC TGCAGACTTGGGTCCTCTGAAACCGGA TGGCGT TTGGCGAGGACTAG

tgt ga tgcaa at cg ct ca ga ga gcat tagt ta tca cc cg ccta ct ca ag ga gg tc ga ga gc gg cgt aa aa cgat ga aa tc tcccgg tt tg gct tc ccaa $\mathrm{AP}-1$ Sp1

ccaltaagtcagagtcccacagagggagaagctgccagcaccaggca taaacacagccttccacacttcgggttatcg tgcgggggctgggctgggctc Sp1

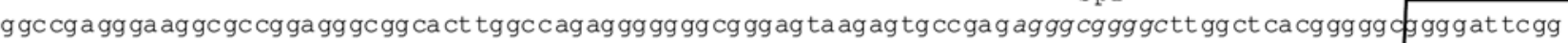
E1-B atg gcg ctt gac cct cta ggc gcc gtc gtc ctg cag agc atc atg gcg cta agc ggt cga ctg gca ttg gcc gcg .... Intron1.

ctc aga ctg tgg ggt ccg gga g ...3,617 bp... gt ggg aga agg ccc ata tcc ctc tgt gtg gga ggc ttt gga ggt

GCC tca ggc gga gga agc agt gag aag aag ttt tct ctg cag gat gta gct gag ctg ctt cgg acc aga gcc tgc Met78

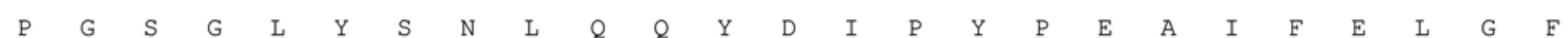
CCA GGG AGC GGC CTC TAC AGC AAC CTT CAG CAG TAT GAC ATC CCG TAC CCT GAA GCC ATC TTT GAA CTT GGC TTT

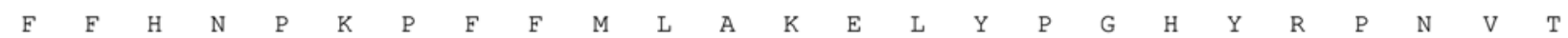
TTC TTT CAC AAC CCC AAG CCC TTT TTC ATG TTG GCC AAG GAG CTG TAC CCT GGG CAC TAC AGG CCC AAT GTC ACT

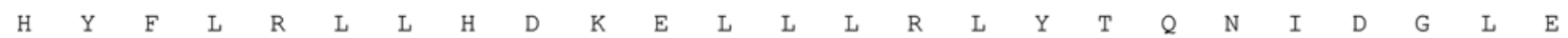
CAC TAC TTC CTG AGg CTC CTC CAC GAC AAg GAG CTG CTT CTG CGg CTC TAT ACA CAG AAC ATC GAC GGG CTT GAG

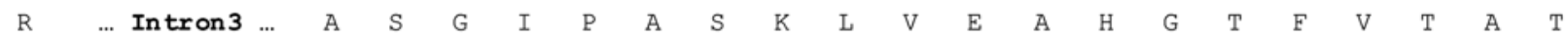
AGA G ... 1,195 bp ... CA TCT GGG ATC CCT GCC TCA AAG CTG GTT GAA GCC CAC GGG ACC TTT GTA ACA GCT ACA $\begin{array}{lllllllllllllllllllllll}C & T & V & C & R & R & S & F & P & G & E & D & I & W & \text { Intron4 } & \text { In } & A & D & V & M & A & D & R\end{array}$ TGG ... 6,703 bp ... GCT GAT GTG ATG GCG GAC AGG $\begin{array}{lllllllllllllllllllllllllllllll}\mathrm{V} & \mathrm{P} & \mathrm{R} & \mathrm{C} & \mathbf{A} & \mathrm{V} & \mathrm{C} & \mathrm{T} & \mathrm{G} & \mathrm{V} & \mathrm{V} & \mathrm{K} & \mathrm{P} & \mathrm{D} & \mathrm{I} & \mathrm{V} & \mathrm{F} & \mathrm{F} & \mathrm{G} & \mathrm{E} & \mathrm{Q} & \mathrm{L} & \mathrm{P} & \mathrm{A} & \mathrm{R}\end{array}$ GTG CCC CGC TGC GCT GTC TGT ACT GGC GTT GTG AAA CCC GAC ATT GTG TTC TTT GGG GAG CAG CTG CCT GCA AGG

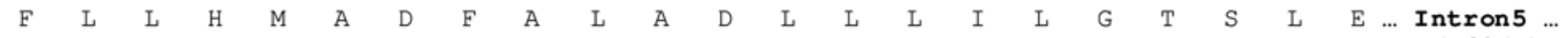
TTC CTA CTC CAT ATG GCT GAC TTC GCT TTG GCA GAT CTG CTA CTC ATT CTT GGG ACC TCC CTG GAG ... 4, 304 bp

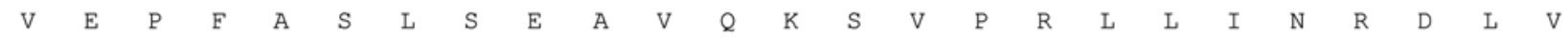
GTG GAG CCT TTT GCC AGC TTG TCT GAA GCA GTA CAG AAA TCA GTG CCC CGA CTG CTC ATC AAT CGA GAC TTG GTG

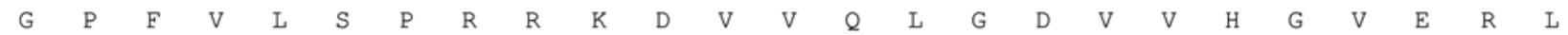
GgG CCG TTC GTT CTG AGT CCT CGA AgG AAA GAT GTG GTC CAg CTA GgG GAT GTA GTT CAT GGT GTG GAA AGg CTG

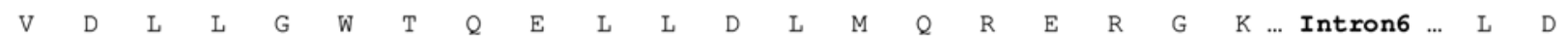
GTG GAC CTC CTG GGG TGG ACA CAA GAA CTG CTG GAT CTT ATG CAG CGG GAA CGT GGC AAG ... 891 bp ... CTG GAT G $Q \quad D \quad R \quad$ *

GGA CAG GAC AGA TAA gactatggcttcttcacctggggaagtcacacagcagatcatcctatgtccagcaagact tcatgcctgaagacagctc caa ca cgt tt acaa acat ga ac ca ga ccacaa ca t gt gg cctg ga ca gt gg tcct cc ga gg ct g cctt tg ga aa gg ct ga ccag ggat gt ct a cc ct tg

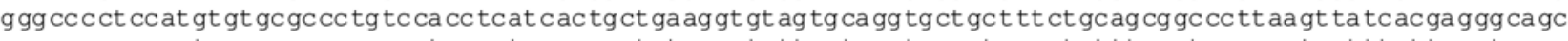
a ca gcacg cc cg tcgccagg caggcgatgcactagggcaatctagca tg ttga tcgg taaagtggcatct ttaactacaaca tcat ttct tgcatgaaa taact tagt at aaaacttggc

Figure 1 (continued). (B) The 1,473 bp murine Sirt3 mRNA has an open reading frame of 774 bp and encodes a 257-aa protein (cytoplasmic SIRT3) with a predictive molecular weight of $28.8 \mathrm{kDa}$ and an isoelectric point of 5.82. The 5' flanking genomic region, which precedes the Sirt3 open reading frame, revealed putative transcription factor binding sites for AML-1a, STATx, GATA, SP1 and AP1. CpG islands were not identified. The 3 ' flanking region was shown to contain the eukaryotic polyadenylation consensus signal AAATAAA 373 bp downstream of the termination of translation signal TAA.

for bootstrap statistical support, horizontal values indicate the percent divergence figures between two pairs of sequences (Fig. 5).

\section{Results}

Identification of cDNAs encoding murine Sirt3. Homology searches of the EST database at NCBI (National Center for
Biotechnology Information) with the yeast SIR2 protein sequence (GenPept P06700) yielded 10 mRNA sequences of variable length: GenBank AF299338.1 (1,473 bp), AF299339.1 (1,428 bp), AK075861.1 (1,385 bp), AK086910.1 (2,527 bp), BC025878.1 (1,372 bp), BF681854.1 (664 bp), CB953804.1 (601 bp), CT010402.1 (774 bp), EU886466.1 (1,406 bp) and FJ621493.1 (1,011 bp). Clone AF299338.1, which contained the murine Sirt3 mRNA reference sequence was then used 


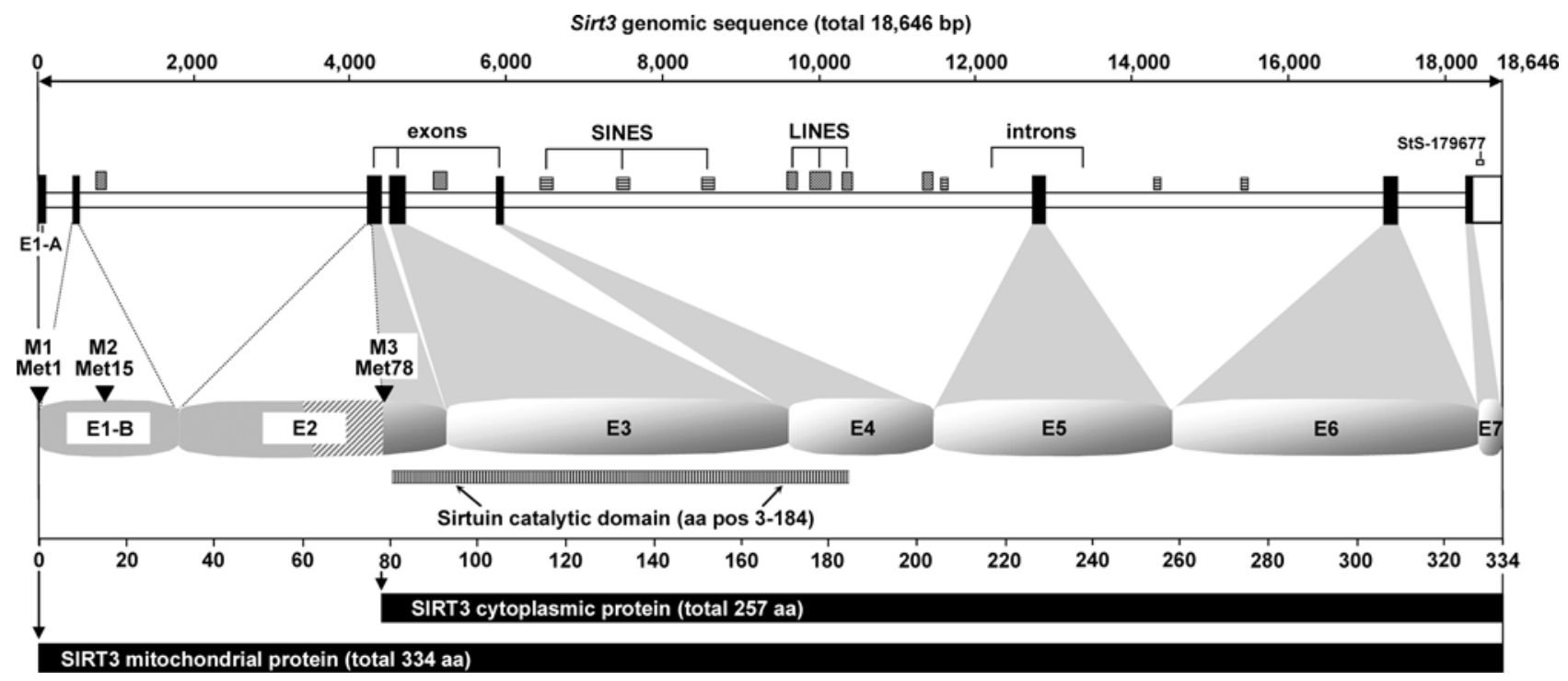

Figure 2. Genomic organization of the murine Sirt3 gene. The genomic organization of the $18.6 \mathrm{~kb}$ long Sirt3 gene, which includes the relative position of exons and introns is shown. Repetitive sequences, known as short and long interspersed nuclear elements (SINEs and LINEs) are indicated. The 3 ' untranslated flanking region of exon 7 contains marker STS-179677. The sirtuin catalytic domain overlaps the SIRT3 protein region that is encoded by exons $2-4$ (aa positions 3-184).

for the identification of the murine Sirt3 genomic clone. The authenticity of its insert was confirmed by DNA cycle sequencing. Sequences flanking the 5' and 3' ends of the Sirt3 open reading frame were identified from the Sirt3 murine genomic clone BAC RP23-114A6. Characterization of the 5 ' flanking genomic region, which precedes the Sirt3 open reading frame, revealed a number of putative transcription factor binding sites for AML-1a, STATx, GATA, SP1 and AP1. However, their biological relevance awaits still to be investigated experimentally. $\mathrm{CpG}$ islands were not identified (Fig. 1).

The 1,473-bp murine Sirt3 mRNA has an open reading frame of $774 \mathrm{bp}$ and encodes a 257 -aa protein (cytoplasmic SIRT3) with a predictive molecular weight of $28.8 \mathrm{kDa}$ and an isoelectric point of 5.82 (Fig. 1B). Lately, a 1,406-bp murine SIRT3 splice variant that encodes a 334 -aa mitochondrial precursor protein with a molecular weight of $36.6 \mathrm{kDa}$ and an isoelectric point of 7.19 has been reported (18) (Fig. 1A). Within exon 1B (E 1-B) translation may start in position Met1 or Met15 (Fig. 1A) $(18,36)$. Fluorescence in situ hybridization analysis identified a single genomic locus for murine Sirt3 gene on chromosome 7F4 and is neighboured by the Ric8 and PSMD13 genes (Fig. 3). Translational stop codons in all reading frames precede the murine Sirt3 open reading frame. The 3' flanking region was shown to contain the eukaryotic polyadenylation consensus signal AAATAAA $373 \mathrm{bp}$ downstream of the termination of translation signal TAA (Fig. 1). The genomic organization of the murine Sirt3 gene is shown in Fig. 2.

Identification and characterization of the murine Sirt3 genomic locus. The murine Sirt3 genomic clone was obtained from an arrayed murine BAC genomic library from the RZPD German Resource Center for Genome Research (Berlin, Germany) after in silico screening with the murine Sirt3 cDNA (GenBank clone AF299338.1), which was shown to contain the full-length murine Sirt3 coding cDNA sequence. BAC clone RP23-114A6 was identified to contain inserts with an average size of $\sim 120 \mathrm{~kb}$ in the $11.6-\mathrm{kb}$ vector pBACe3.6, which included the murine Sirt3 genomic sequence. BAC genomic DNA was prepared according to published protocols (31) and the Sirt3 insert was confirmed by cycle sequencing (32). Genomic sequence comparison analyses with the BLAST algorithm helped us with the identification of mouse chromosome 7 genomic contig GenBank AC107815.11. We have used this sequence for the determination of Sirt3 introns and exon/intron boundaries (Table I). The murine Sirt3 gene spans a region of 18,646 bp (Fig. 2). Determination of the exon-intron splice junctions established that the gene Sirt3 is encoded by 7 exons ranging in size from $101 \mathrm{bp}$ (exon 4) to 420 bp (exon 7). In reference to the initial report (37) on the intron sizes we have identified several discrepancies, which are shown in Table I (the intron sizes that have been reported by Yang et al are shown in curly brackets, while the intron sizes that we have identified are shown in bold. Within introns 1, 3, 4 and 5 we identified an accumulation of interspersed repetitive elements, SINEs (short interspersed nuclear elements) and LINEs (long interspersed nuclear elements) (Fig. 2). Additionally, we have identified marker STS-179677 within the untranslated proportion of murine Sirt3 exon 7. The sirtuin catalytic domain, which is highly conserved in all members of mammalian sirtuins that have been described so far as well as in their Sir2 yeast ancestor protein, is found between amino acid residues 3 and 184 of the 257-aa protein (i.e., within aa position 80 and 261 of the 334-aa protein and is encoded by exons 2 and 4 (Fig. 2).

Murine Sirt3 is a single copy gene. Both sequencing and results obtained by electronic PCR of BAC clone RP23-114A6 identified marker STS-179677 within exon 7 of the Sirt3 genomic sequence (Fig. 2). Our fluorescence in situ hybridization studies localized Sirt3 to chromosome 7F4. These 


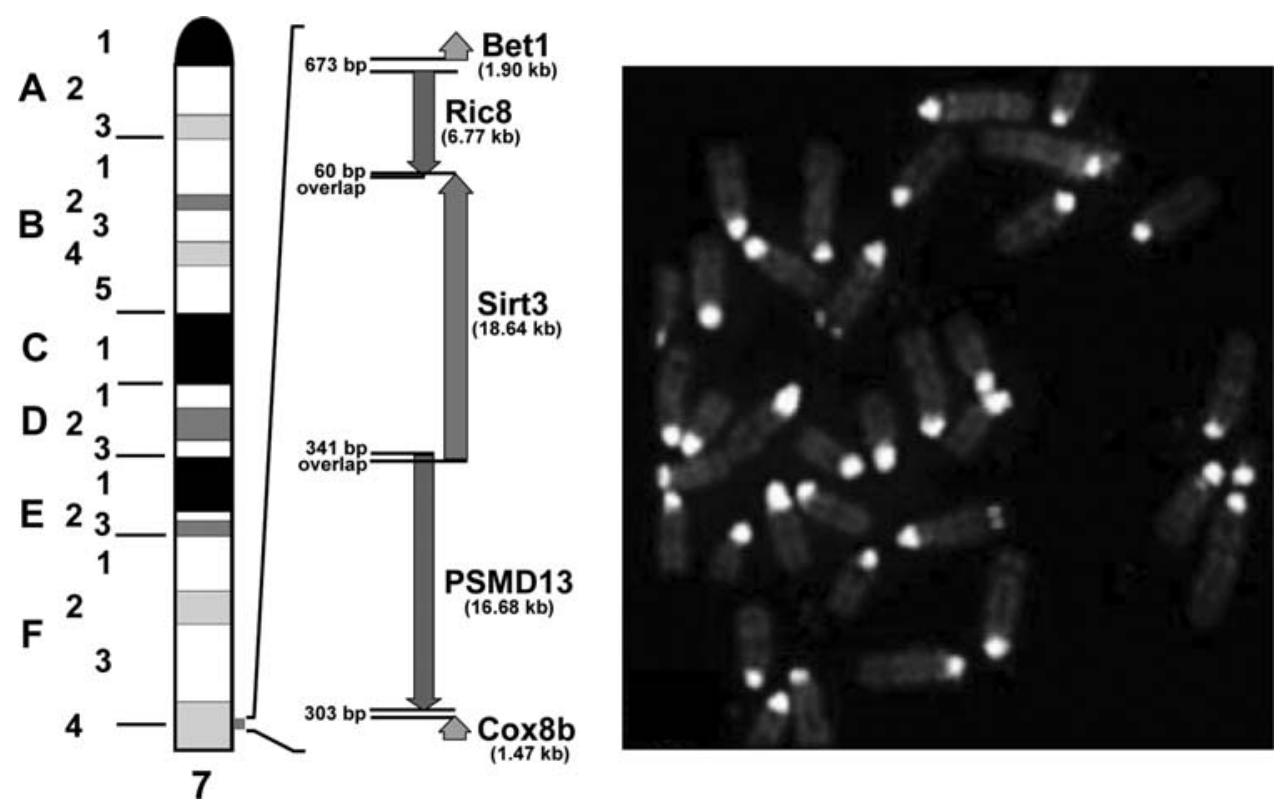

Figure 3. Chromosomal mapping of the murine Sirt3 gene. Right panel, fluorescence in situ hybridization of BAC clone RP23-114A6 to mouse chromosome 7F4. Left panel, chromosome 7 idiogram, which illustrates the chromosomal position of the Sirt 3 gene, which is being closely neighboured by the Ric 8 and PSMD13 genes.

data, together with the results obtained by electronic PCR and the already known location of the STS markers listed above, indicated one single site of hybridization of Sirt3 on murine metaphase chromosomes and its specific localization on chromosome 7F4 (Fig. 3), and is therefore located within autosomal gene synteny groups in mouse and man (Fig. 4).

Sirt3 expression analyses. In silico approximate expression patterns have been inferred with the UniGene EST profile viewer, which is hosted by the NCBI homepage and which suggested the strongest expression of murine Sirt3 in tissues that are highly metabolically active such as brown adipose tissue, muscle, liver, the kidneys, heart and brain $(13,19,20)$.

Phylogenetic analyses and pairwise sequence comparisons. We have screened the expressed sequence tag database (NCBI) with the yeast Sir2 protein sequence and identified the SIRT3 protein sequences in various different species. A consensus evolutionary tree was obtained using an alignment of yeast SIR2 with a selection of different mammalian and nonmammalian SIRT3 homologs (Fig. 5). The accession numbers of the sequences that have been used in this phylogenetic analysis were as follows: yeast Sir2 (GenPept P06700), Homo sapiens (GenPept NP_001017524.1), Mus musculus (GenPept CAJ18608.1), Monodelphis domestica (GenPept XP_001380188.1), Gallus gallus (GenPept XP_420920.2), Dario rerio (GenPept CAQ14722.1), Takifugu rubripes (ENSTRUP00000031533) and Xenopus tropicalis (GenPept NP_001120529.1) (Fig. 5A).

Yeast Sir2 (GenPept P06700), Mus musculus SIRT3 (GenPept CAJ18608.1), Mus musculus SIRT2 (GenPept AAH21439.1), Mus musculus SIRT1 (GenPept Q923E4), Mus musculus SIRT4 (GenPept XP_993153), Mus musculus SIRT7 (GenPept NP_694696.2), Mus musculus SIRT6 (GenPept AAH52763.1), Mus musculus SIRT5 (GenPept AAH31770.1), Mus musculus HDAC1 (GenPept CAQ

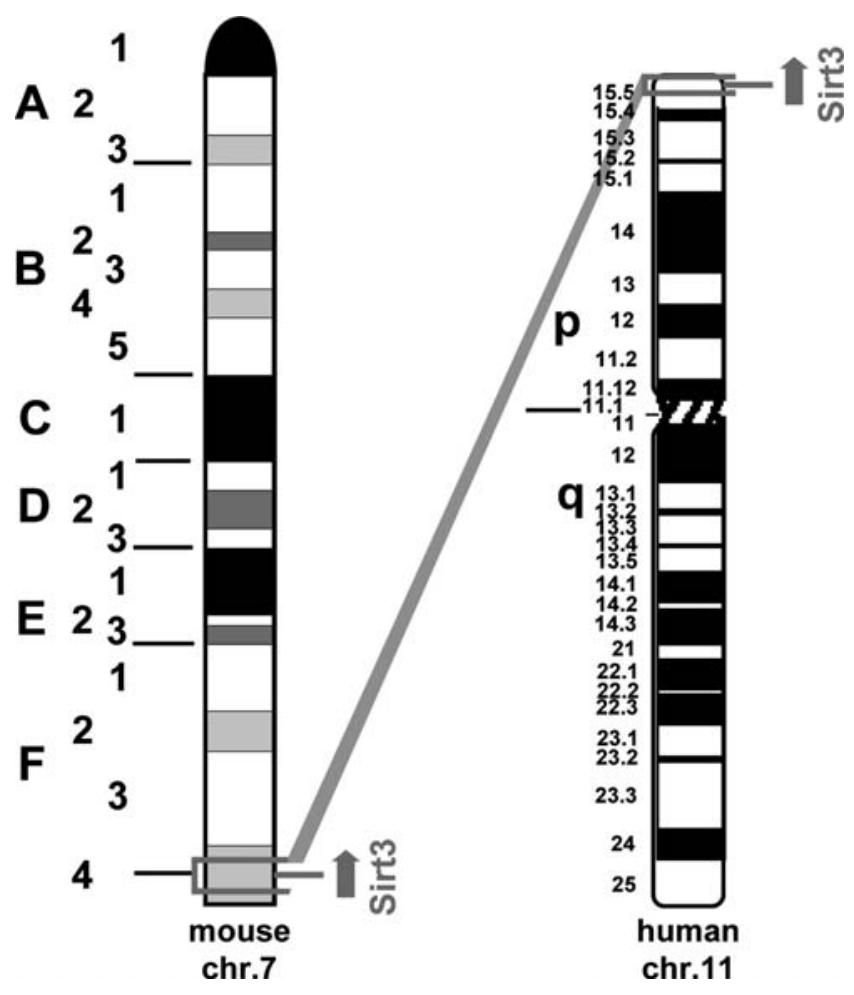

Figure 4. The Sirt3 gene is located within autosomal gene synteny groups in mouse and man (mouse Sirt3 is located on chromosome 7F4 and human Sirt3 is located on chromosome 11p15.5) and is therefore syntenically conserved.

51569.1), Mus musculus HDAC2 (GenPept AAI38518.1), Mus musculus HDAC3 (GenPept AAF36425.1), Mus Musculus HDAC4 (GenPept AAH66052.1), Mus musculus HDAC5 (GenPept CAX15947.1), Mus musculus HDAC6 (GenPept AAH41105.1), Mus musculus HDAC7 (GenPept AAH57332.1), Mus musculus HDAC8 (GenPept AAH 


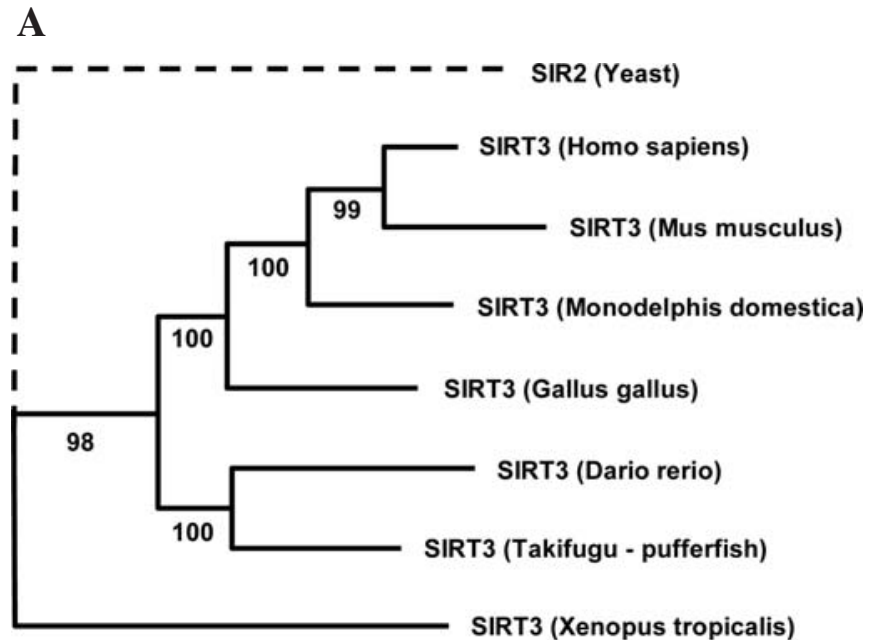

B

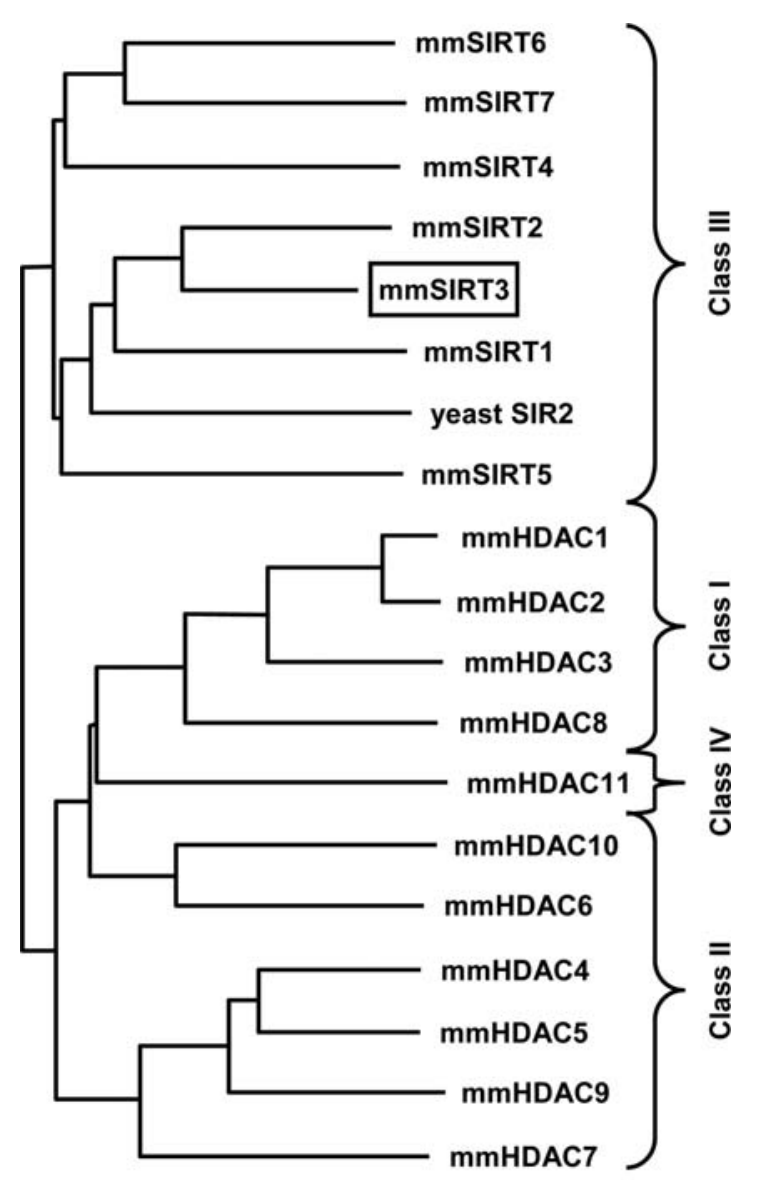

10.00

Figure 5. (A) Consensus evolutionary tree on the basis of an alignment of SIRT3 from different species together with their common ancestor protein, yeast SIR2. The numbers denote the bootstrap values. (B) Evolutionary tree revealing the position of murine SIRT3 in the context of the other known class I-IV histone deacetylases. The indicated numbers describe the substitutions per 100 residues.

61257.1), Mus musculus HDAC9 (GenPept AAH98187.1), Mus musculus HDAC10 (GenPept AAH64018.1) and Mus musculus HDAC11 (GenPept AAH16208.1) (Fig. 5B).

\section{Discussion}

Despite the fact that murine and human SIRT3 share a high degree of protein sequence conservation, there are some remarkable differences between the two proteins: while human or any other mammalian SIRT3 that has been reported so far, does not have a methionine in position 15, murine SIRT3 does and in fact, it appears that varying amounts of the M1 variant (starting at Met1) and the M2 variant (starting at Met15) may be detected depending on the cell type that is being examined and it has been postulated that the choice of the murine SIRT3 translation initiation site (Met1 vs. Met15) may serve as a regulatory element in the expression of proteins with variations in the extent of proteolytic processing in mitochondria. A third variant of murine SIRT3 is the Met78 (M3) variant, which is a non-mitochondrial protein that may be detected in the cytosol and in the cell nucleus. This M3 variant is being detected at lower levels when compared to the M1 and M2 murine SIRT3 variants $(18,36)$.

Mouse SIRT3 (mSIRT3) was first cloned by Yang et al in the year 2000 (37). Back then it was termed mSIR2L3 (mouse SIR2 like protein 3) and reported as a 257 amino acid protein. With our highest respect as to the report of Yang et al, our report adds some corrections and important additions to the data reported in the year 2000. We confirm the genomic localization of murine Sirt3 on mouse chromosome 7F4 and would like to add that the genomic sequence of murine Sirt3 overlaps with the Ric 8 gene at its 3 ' end and with the PSMD13 gene at its $5^{\prime}$ end, which are both located on the complementary strand. The orientation of the Sirt3 gene in the mouse is towards the centromere (Fig. 3). On the same strand, Sirt 3 is being neighboured by the Bet 1 gene $(1,90 \mathrm{~kb}$ downstream of its centromeric end) and by the Cox $8 b$ gene (16,99 $\mathrm{kg}$ upstream of its telomeric end). In addition, we identified marker STS-179677 to be located within the untranslated region of exon 7 (Figs. 2 and 3). The Sirt3 gene contains two alternative exons $(\mathrm{E}-1 \mathrm{~A}$, which is not translated and $\mathrm{E}-1 \mathrm{~B}$, which contains two translational start sites Met1 and Met15) and six constant exons (E2-E7). These exons range in size from $101 \mathrm{bp}$ (exon 4) to $420 \mathrm{bp}$ (exon 7; Table I). As to the report of Yang et al (37) we have identified a number of discrepancies with regard to the intron sizes that have been reported (Table I). Murine Sirt3 is a single-copy gene that spans a region of 18,646 kb. Within introns $1,3,4$ and 5 we identified an accumulation of interspersed repetitive elements, which consist of Alu or KpnI and BamH1 repeats as representative examples of short and long interspersed nuclear elements, known as SINEs (Alu repeats) and LINEs (KpnI and BamH1 repeats) (38). The sirtuin catalytic domain, which is found between amino acid residues 3 and 184 of the 257-aa protein (i.e. within aa position 80 and 261 of the 334-aa protein) is encoded by exons 2 and 4 (Fig. 2).

SIRT3 has been reported to be important in the context of cellular respiration and metabolism. Calorie restriction, stress and exercise go along with elevated levels of SIRT3 $(20,22,23)$. In addition, an overexpression of SIRT3 may also be observed in some forms of cancer, such as node-positive breast cancer (24) or in acute myeloid leukemia (unpublished data). In addition, SIRT3 and SIRT1 are known to interact with the heat-shock protein HSP70 (39), which is relevant in relation 
to aging-related disease such as Parkinson's and Alzheimer's disease and Huntington's chorea (40).

The sirtuins are known to be essential regulators of longevity and elements that are essential in the regulation of transcription, apoptosis and cellular response to stress. Also, they are known to be important in the regulation of cellular metabolism and in the pathogenesis of cancer and degenerative disease. Therefore, the sirtuins are gaining continuously increasing importance as molecular targets in the development of novel epigenetic therapeutics (41-43). From within the 7 known mammalian sirtuins, SIRT3 has recently come forward and out of the shadow of SIRT1 revealing strong effects in relation to cellular stress $(22,44)$, cell growth control and in cell cycle regulation (45) and especially on energy metabolism in the context of calorie restriction $(26,46,47)$.

\section{Acknowledgments}

This work was supported by grants from the Deutsche José Carreras Leukämie-Stiftung e. V. (DJCLS R 09/20).

\section{References}

1. Mahlknecht U and Hoelzer D: Histone acetylation modifiers in the pathogenesis of malignant disease. Mol Med 6: 623-644, 2000.

2. Frye RA: Characterization of five human cDNAs with homology to the yeast SIR2 gene: Sir2-like proteins (sirtuins) metabolize NAD and may have protein ADP-ribosyltransferase activity. Biochem Biophys Res Commun 260: 273-279, 1999.

3. Frye RA: Phylogenetic classification of prokaryotic and eukaryotic Sir2-like proteins. Biochem Biophys Res Commun 273: 793-798, 2000.

4. Landry J, Sutton A, Tafrov ST, et al: The silencing protein SIR2 and its homologs are NAD-dependent protein deacetylases. Proc Natl Acad Sci USA 97: 5807-5811, 2000.

5. Tanner KG, Landry J, Sternglanz R and Denu JM: Silent information regulator 2 family of NAD-dependent histone/protein deacetylases generates a unique product, 1-O-acetyl-ADPribose. Proc Natl Acad Sci USA 97: 14178-14182, 2000.

6. Brachmann CB, Sherman JM, Devine SE, Cameron EE, Pillus L and Boeke JD: The SIR2 gene family, conserved from bacteria to humans, functions in silencing, cell cycle progression, and chromosome stability. Genes Dev 9: 2888-2902, 1995.

7. Saunders LR and Verdin E: Sirtuins: critical regulators at the crossroads between cancer and aging. Oncogene 26: 5489-5504, 2007.

8. Zschoernig B and Mahlknecht U: SIRTUIN 1: regulating the regulator. Biochem Biophys Res Commun 376: 251-255, 2008.

9. Zhao K, Chai X, Clements A and Marmorstein R: Structure and autoregulation of the yeast Hst2 homolog of Sir2. Nat Struct Biol 10: 864-871, 2003 .

10. Haigis MC, Mostoslavsky R, Haigis KM, et al: SIRT4 inhibits glutamate dehydrogenase and opposes the effects of calorie restriction in pancreatic beta cells. Cell 126: 941-954, 2006.

11. Michishita E, McCord RA, Berber E, et al: SIRT6 is a histone $\mathrm{H} 3$ lysine 9 deacetylase that modulates telomeric chromatin. Nature 452: 492-496, 2008

12. Nakagawa T, Lomb DJ, Haigis MC and Guarente L: SIRT5 Deacetylates carbamoyl phosphate synthetase 1 and regulates the urea cycle. Cell 137: 560-570, 2009.

13. Schwer B, North BJ, Frye RA, Ott M and Verdin E: The human silent information regulator (Sir)2 homologue hSIRT3 is a mitochondrial nicotinamide adenine dinucleotide-dependent deacetylase. J Cell Biol 158: 647-657, 2002.

14. Schwer B, Bunkenborg J, Verdin RO, Andersen JS and Verdin E: Reversible lysine acetylation controls the activity of the mitochondrial enzyme acetyl-CoA synthetase 2. Proc Natl Acad Sci USA 103: 10224-10229, 2006.
15. Michishita E, Park JY, Burneskis JM, Barrett JC and Horikawa I: Evolutionarily conserved and nonconserved cellular localizations and functions of human SIRT proteins. Mol Biol Cell 16: 4623-4635, 2005.

16. Haigis MC and Guarente LP: Mammalian sirtuins - emerging roles in physiology, aging, and calorie restriction. Genes Dev 20: 2913-2921, 2006.

17. Lavu S, Boss O, Elliott PJ and Lambert PD: Sirtuins - novel therapeutic targets to treat age-associated diseases. Nat Rev Drug Discov 7: 841-853, 2008.

18. Cooper HM, Huang JY, Verdin E and Spelbrink JN: A new splice variant of the mouse SIRT3 gene encodes the mitochondrial precursor protein. PLoS ONE 4: e4986, 2009.

19. Onyango P, Celic I, McCaffery JM, Boeke JD and Feinberg AP: SIRT3, a human SIR2 homologue, is an NAD-dependent deacetylase localized to mitochondria. Proc Natl Acad Sci USA 99: 13653-13658, 2002.

20. Shi T, Wang F, Stieren E and Tong Q: SIRT3, a mitochondrial sirtuin deacetylase, regulates mitochondrial function and thermogenesis in brown adipocytes. J Biol Chem 280: 13560-13567, 2005.

21. Lombard DB, Alt FW, Cheng HL, et al: Mammalian Sir2 homolog SIRT3 regulates global mitochondrial lysine acetylation. Mol Cell Biol 27: 8807-8814, 2007.

22. Sundaresan NR, Samant SA, Pillai VB, Rajamohan SB and Gupta MP: SIRT3 is a stress-responsive deacetylase in cardiomyocytes that protects cells from stress-mediated cell death by deacetylation of Ku70. Mol Cell Biol 28: 6384-6401, 2008.

23. Palacios OM, Carmona JJ, Michan S, et al: Diet and exercise signals regulate SIRT3 and activate AMPK and PGC-1alpha in skeletal muscle. Aging 1: 771-783, 2009.

24. Ashraf N, Zino S, Macintyre A, et al: Altered sirtuin expression is associated with node-positive breast cancer. Br J Cancer 95: 1056-1061, 2006.

25. Hallows WC, Lee S and Denu JM: Sirtuins deacetylate and activate mammalian acetyl-CoA synthetases. Proc Natl Acad Sci USA 103: 10230-10235, 2006.

26. Schlicker C, Gertz M, Papatheodorou P, Kachholz B, Becker CF and Steegborn C: Substrates and regulation mechanisms for the human mitochondrial sirtuins Sirt3 and Sirt5. J Mol Biol 382: 790-801, 2008.

27. Yang Y, Cimen H, Han MJ, et al: NAD ${ }^{+}$-dependent deacetylase SIRT3 regulates mitochondrial protein synthesis by deacetylation of the ribosomal protein MRPL10. J Biol Chem 285: 7417-7429, 2010.

28. Ahn BH, Kim HS, Song S, et al: A role for the mitochondrial deacetylase Sirt3 in regulating energy homeostasis. Proc Natl Acad Sci USA 105: 14447-14452, 2008.

29. Bellizzi D, Dato S, Cavalcante P, et al: Characterization of a bidirectional promoter shared between two human genes related to aging: SIRT3 and PSMD13. Genomics 89: 143-150, 2007.

30. Bellizzi D, Rose G, Cavalcante P, et al: A novel VNTR enhancer within the SIRT3 gene, a human homologue of SIR2, is associated with survival at oldest ages. Genomics 85: 258-263, 2005.

31. Birnboim HC and Doly $\mathrm{J}$ : A rapid alkaline extraction procedure for screening recombinant plasmid DNA. Nucleic Acids Res 7: 1513-1523, 1979

32. Mahlknecht U, Hoelzer D and Bucala R: Sequencing of genomic DNA. Biotechniques 27: 406-408, 1999.

33. Altschul SF, Madden TL, Schaffer AA, et al: Gapped BLAST and PSI-BLAST: a new generation of protein database search programs. Nucleic Acids Res 25: 3389-3402, 1997.

34. Wilbur WJ and Lipman DJ: Rapid similarity searches of nucleic acid and protein data banks. Proc Natl Acad Sci USA 80: 726-730, 1983.

35. Thompson JD, Higgins DG and Gibson TJ: CLUSTAL W: improving the sensitivity of progressive multiple sequence alignment through sequence weighting, position-specific gap penalties and weight matrix choice. Nucleic Acids Res 22: 4673-4680, 1994

36. Cooper HM and Spelbrink JN: The human SIRT3 protein deacetylase is exclusively mitochondrial. Biochem J 411: 279-285, 2008.

37. Yang YH, Chen YH, Zhang CY, Nimmakayalu MA, Ward DC and Weissman S: Cloning and characterization of two mouse genes with homology to the yeast Sir2 gene. Genomics 69: 355-369, 2000 
38. Singer MF, Thayer RE, Grimaldi G, Lerman MI and Fanning TG: Homology between the KpnI primate and BamH1 (M1F-1) rodent families of long interspersed repeated sequences. Nucleic Acids Res 11: 5739-5745, 1983.

39. Law IK, Liu L, Xu A, et al: Identification and characterization of proteins interacting with SIRT1 and SIRT3: implications in the anti-aging and metabolic effects of sirtuins. Proteomics 9: 2444-2456, 2009.

40. Soti $\mathrm{C}$ and Csermely P: Chaperones and aging: role in neurodegeneration and in other civilizational diseases. Neurochem Int 41: 383-389, 2002

41. Dali-Youcef N, Lagouge M, Froelich S, Koehl C, Schoonjans K and Auwerx J: Sirtuins: the 'magnificent seven', function, metabolism and longevity. Ann Med 39: 335-345, 2007.

42. Michan S and Sinclair D: Sirtuins in mammals: insights into their biological function. Biochem J 404: 1-13, 2007.
43. Voelter-Mahlknecht S and Mahlknecht U: The sirtuins in the pathogenesis of cancer. Clin Epigenetics 1: 1-13, 2010.

44. Haigis MC and Sinclair DA: Mammalian sirtuins: biological insights and disease relevance. Annu Rev Pathol 5: 253-295, 2010.

45. Allison SJ and Milner J: SIRT3 is pro-apoptotic and participates in distinct basal apoptotic pathways. Cell Cycle 6: 2669-2677, 2007.

46. Dransfeld C, Alborzinia H, Wölfl S and Mahlknecht U: Continuous multiparametric monitoring of cell metabolism in response to transient overexpression of the sirtuin deacetylase SIRT3. Clinical Epigenetics 1: 1-6, 2010.

47. Dransfeld CL, Alborzinia H, Wolfl S and Mahlknecht U: SIRT3 SNPs validation in 640 individuals, functional analyses and new insights into SIRT3 stability. Int J Oncol 36: 955-960, 2010. 